\title{
QTL analysis for stomatal density and size in wheat spike organ
}

\author{
Shuguang Wang', Fanfan Dong1, Daizhen Sun ${ }^{1 *}$, Yaoyu Chen ${ }^{1}$, Xue Yan ${ }^{1}$, Ruilian Jing ${ }^{2}$ \\ ${ }^{1}$ College of Agronomy, Shanxi Agricultural University, Taigu, 030801, P.R. China, ${ }^{2}$ Institute of Crop Science, Chinese Academy of Agricultural \\ Sciences, Beijing 100081, P.R.China
}

\author{
A B S T R A C T
}

\begin{abstract}
Plant changes its own photosynthetic rate and transpiration rate through regulating stomatal aperture, stomatal density and stomatal distribution. In this study, stomatal density, length and width of wheat spike organs, including palea, lemma and glume, at the third day after flowering were investigated, using a wheat doubled haploid population from a cross of Hanxuan10 and Lumai 14 in 2012 and 2013. And quantitative trait loci (QTL) of the above three traits were analyzed. There were stomata in the abaxial surface of palea, lemma and glume, but not in the adaxial surface for DH lines and their parents. A total of fourteen additive QTLs for those traits were identified. On the marker interval Xgwm291-Xgwm410-WMC340 on chromosome 5A, $Q_{M L s d}-5 A$ for stomatal density at middle of lemma and $Q_{D G s d}-5 A$ for stomatal density at down of glume, and $Q_{A G S}-5 A$ for stomatal length at apex of glume were detected in 2012 and 2013 , but with opposite direction of additive effect. In the previous study, Qsd-5A.3 and Qsd-5A.4 for stomatal density of wheat leaf, and $Q s /-5 A$. 1 for stomatal length of wheat leaf were also detected at the same marker region, and also with opposite direction of additive effect. These findings provided genetic basis for significantly negative correlation between stomatal density and length for wheat leaf and spike organs, but also implied stomatal density and length for wheat leaf and spike organs may be governed by the same or pleiotropic genes.
\end{abstract}

Keywords: Stomatal density; Stomatal length; Stomatal width; Spike organ; QTL

\section{INTRODUCTION}

Non-foliar green organs in wheat are another major source of photosynthesis, except for leaf organ. Specially, spike organs at the top of the canopy are conducive to the interception of photosynthetic $\mathrm{CO}_{2}$ (Zhang et al., 2006). The photosynthetic product from spike organs is transported directly to the grain without waste (Zhang et al., 2008). Changes of physiological indexes reflecting the level of photosynthetic capacity, such as stomatal conductance, net photosynthetic rate, and transpiration rate, are mainly implemented through the stomata. Plant can change its own photosynthetic rate and transpiration rate through regulating stomatal aperture, stomatal density and stomatal distribution, when it is stressed by biotic or abiotic factors (Meidner 1986).

It was showed that stomata existed on all green parts of spike organs. Teare et al. (1971) found that there were stomata in the abaxial surface of glume, lemma, palea and awn, but not in the adaxial surface. Ziegler-Jons et al.
(1989) recognized that there were stomata only in the adaxial surface of glume, in the abaxial and adaxial base of lemma in wheat. Zhang et al. (2006) thought there were stomata on different parts of spike organs. Obviously, there are different views about stomatal distribution of spike organs in wheat. So far, there were many reports of genetic analysis of traits related to stomata in leaf, for example, in rice, two QTLs for adaxial stomatal frequencies and two QTLs for abaxial stomatal frequencies in the middle part of the top fully expanded leaves were identified (Ishimaru et al., 2001a). Ten QTLs for stomatal density and four QTLs for size were detected across growth stages and leaf surfaces (adaxial and abaxial) (Laza et al., 2010). In wheat, twenty additive QTLs and 19 pairs of epistatic QTLs for stomatal density, stomatal length and width of leaf were identified under drought stress, the other twenty QTLs and 25 pairs epistatic QTLs were obtained under well-water at the heading, flowering, and mid- and late grain filling stages (Wang et al., 2016). However, there have not been about genetic analysis of traits related to stomata in spike organs. In this study, stomatal density, stomatal length and 
width in palea, apex of lemma, middle of lemma, apex of glume, middle of glume, down of glume were measured and QTLs for those traits were analyzed. The purpose was to gain insights into the changing pattern and molecular basis of stomatal density and size in spike organs.

\section{MATERIALS AND METHODS}

\section{Plant material and field design}

The wheat DH population, derived from a Hanxuan 10 (H10) $\times$ Lumai 14 (L14) cross, comprised of 150 lines (Jing et al., 1999). All 150 lines and parents were grown at the experimental farm $\left(37^{\circ} 25^{\prime} \mathrm{N}, 112^{\circ} 35^{\prime} \mathrm{E}, 799.6\right.$ m.a.s.l.) of Shanxi Agricultural University in 2012 and 2013. The experimental soil was sandy with, soil organic matter of $16.7 \mathrm{~g} \mathrm{~kg}-1$, total nitrogen of $2.9 \mathrm{~g} \mathrm{~kg}-1$, available phosphorus of $11.2 \mathrm{mg} \mathrm{kg-1}$, available potassium of $110.7 \mathrm{mg} \mathrm{kg}-1$.The field design consisted of randomized complete blocks with three replications. Each block was a length of $2 \mathrm{~m}$ and a width of $40 \mathrm{~m} .150$ lines and two parents with $0.25 \mathrm{~m}$ between rows and 40 seeds per row were sown. Irrigations with $650 \mathrm{~m}^{3} / \mathrm{hm}^{2}$ were applied at Zadoks growth stages (GS)22, 30, 40 and 65, respectively.

\section{Measurement and calculation of stomatal density and size in spike organs}

Three flowering plants in each line and parents were tagged. The three plants looked like the same. Middle spikes of the three plants were quickly placed into $2 \mathrm{~mL}$ centrifuge tube with FAA solution (alcohol 70\%: acetic acid: formalin=18:1:1) 3 days after anthesis, respectively. Every tube was gently shaken, then placed in a refrigerator at $4 \mathrm{C}^{\circ}$.

After a spike was taken out, the first flower on the base was selected. The surfaces of all parts were cleaned using a degreased cotton ball dipped in alcohol, and then carefully smeared with a thin film of nail varnish on the under epidermis. When dry, the film was peeled from the leaf surface, mounted on a glass slide, and immediately covered with a cover slip.

Five views were selected in palea, apex of lemma, middle of lemma, apex of glume, middle of glume, down of glume, respectively. The numbers of stomata per view were scored, and stomatal lengths and widths were measured under a $40 \mathrm{X}$ objective lens of a photomicroscope fitted with objective and eyepiece micrometers. Stomatal averages of five view areas $\left(\mathrm{S}=\pi \mathrm{r}^{2}, \mathrm{r}=\right.$ view radius) were calculated, and stomatal density was defined as $\mathrm{N} / \mathrm{S}$ (number of stomata $\mathrm{mm}-2)$. Three random stomata per view were randomly selected for measuring lengths and widths which were then meant as the value for each plant.

\section{Statistical analyses and QTL detection}

Analyses of variance (ANOVA) of the data were conducted by the SPSS v.17.0 statistical package to assess the variances among DHLs and to calculate the mean, variance, standard deviation, coefficient of variation, kurtosis and skewness of DHLs for stomatal density and size and differences of the same traits between two years.

QTLs for stomatal density and size were detected using QTL Network 2.0 for composite interval mapping (CIM) of a mixed linear model. Significance levels of $\mathrm{P}<0.001$ and $\mathrm{P}<0.005$ were adopted for identifying additive effects of QTLs. QTLs were named according to the rule of "QTL + trait + chromosome + gene number".

\section{RESULTS}

\section{Stomatal distribution of spike organs in wheat}

There were stomata in the abaxial surface of palea, lemma and glume, but not in the adaxial surface for $\mathrm{DH}$ lines and their parents. For palea, there were two to three rows of stomata at the crease part of the edge of the abaxial surface and were covered by trichome. From the apex to the middle to the base of lemma, the row number of stomata changed from more to one to zero, and the density of stomata also became smaller. More rows of stomata existed at the edge of glume, and from the apex to the base, the row number of stomata became littler, the density of stomata gradually became smaller (Fig. 1).

\section{Phenotypic variation in stomatal density and size in the DHLs and their parents}

Except for apex, middle and down of glume of Lumai14 and down of glume of Hanxuan10 (Fig. 2), differences of stomatal densities for the rest parts of spike organs between

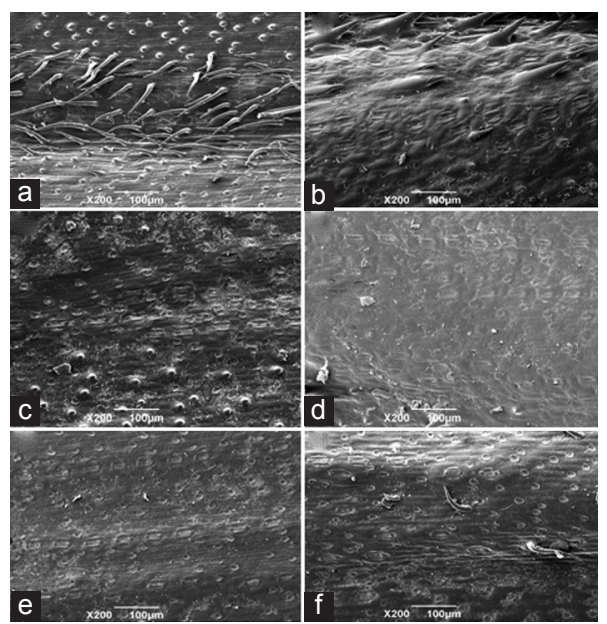

Fig 1. The distribution of stomata on spike organs of wheat. a: Palea, b: Apex of Lemma, c: Middle of Lemma, d: Apex of Glume, e: Middle of Glume, f: Down of Glume. 
Wang, et al.

Table 1: The phenotype of stomatal density, length and width of spike organs in DH population and their parents

\begin{tabular}{|c|c|c|c|c|c|c|c|c|c|c|c|}
\hline Trait & Environment & $\mathrm{H} 10$ & L14 & $t$ & Min. & Mix. & Mean & SD & Skewness & Kurtosis & CV (\%) \\
\hline \multirow[t]{2}{*}{$\mathrm{P}_{\mathrm{SD}}$} & 2012 & $92.785 a / A$ & $81.395 a / A$ & 1.1837 & 71.167 & 111.507 & $88.722 a / A$ & 9.208 & 0.203 & -0.473 & 10.31 \\
\hline & 2013 & $82.556 \mathrm{a} / \mathrm{A}$ & $85.458 a / A$ & 1.8865 & 55.932 & 105.989 & $81.375 \mathrm{~b} / \mathrm{B}$ & 7.885 & 0.029 & 0.507 & 9.79 \\
\hline \multirow[t]{2}{*}{$\mathrm{AL}_{\mathrm{SD}}$} & 2012 & $87.852 \mathrm{a} / \mathrm{A}$ & $76.607 a / A$ & $4.4745^{*}$ & 66.488 & 114.368 & $86.11 \mathrm{a} / \mathrm{A}$ & 8.331 & 0.231 & 0.334 & 9.56 \\
\hline & 2013 & $86.981 \mathrm{a} / \mathrm{A}$ & $84.66 \mathrm{a} / \mathrm{A}$ & $7.6414^{*}$ & 69.788 & 101.781 & $84.270 \mathrm{a} / \mathrm{A}$ & 6.749 & 0.105 & -0.392 & 8.01 \\
\hline \multirow[t]{2}{*}{$\mathrm{ML}_{\mathrm{SD}}$} & 2012 & $79.328 a / A$ & $59.088 a / A$ & 0.0642 & 48.75 & 113.098 & $71.62 \mathrm{a} / \mathrm{A}$ & 10.618 & 0.654 & 1.101 & 14.78 \\
\hline & 2013 & $84.679 a / A$ & $61.754 \mathrm{a} / \mathrm{A}$ & 0.4724 & 48.968 & 99.242 & $67.252 \mathrm{~b} / \mathrm{B}$ & 7.535 & 0.59 & 2.107 & 11.15 \\
\hline \multirow[t]{2}{*}{$\mathrm{AG}_{\mathrm{SD}}$} & 2012 & $88.686 a / A$ & $80.525 a / A$ & 0.8505 & 43.636 & 110.341 & $85.044 a / A$ & 10.488 & -0.374 & 0.832 & 12.27 \\
\hline & 2013 & $87.054 a / A$ & $73.016 \mathrm{~b} / \mathrm{B}$ & 0.3065 & 62.026 & 105.771 & $79.438 \mathrm{~b} / \mathrm{B}$ & 7.857 & 0.464 & 0.507 & 9.89 \\
\hline \multirow{2}{*}{$\mathrm{MG}_{\mathrm{SD}}$} & 2012 & $76.172 \mathrm{a} / \mathrm{A}$ & $76.063 \mathrm{a} / \mathrm{A}$ & $23.372^{*}$ & 50.927 & 111.103 & $77.893 a / A$ & 10.461 & 0.297 & 0.243 & 13.36 \\
\hline & 2013 & $81.069 a / A$ & $60.393 \mathrm{~b} / \mathrm{B}$ & 2.6286 & 51.144 & 93.366 & $73.473 \mathrm{~b} / \mathrm{B}$ & 8.259 & 0.04 & -0.08 & 11.18 \\
\hline \multirow{2}{*}{$\mathrm{DG}_{\mathrm{SD}}$} & 2012 & $72.254 \mathrm{~b} / \mathrm{A}$ & $72.907 a / A$ & $82.2725^{\star \star}$ & 42.439 & 104.356 & $70.367 a / A$ & 10.954 & 0.152 & 0.387 & 15.1 \\
\hline & 2013 & $80.946 a / A$ & $62.026 \mathrm{~b} / \mathrm{A}$ & 3.544 & 39.174 & 104.9 & $68.269 a / A$ & 10.5 & 0.107 & 0.768 & 15.10 \\
\hline \multirow[t]{2}{*}{$P_{S L}$} & 2012 & $47.083 a / A$ & $44.166 a / A$ & 1.089 & 36.25 & 49.625 & $42.933 \mathrm{~b} / \mathrm{A}$ & 2.463 & 0.126 & 0.18 & 5.75 \\
\hline & 2013 & $44.479 a / A$ & $43.125 \mathrm{a} / \mathrm{A}$ & 0.7559 & 37.727 & 47.875 & $43.178 a / A$ & 1.849 & 0.316 & 0.231 & 4.28 \\
\hline \multirow[t]{2}{*}{$\mathrm{AL}_{\mathrm{SL}}$} & 2012 & $48.541 \mathrm{a} / \mathrm{A}$ & $46.77 a / A$ & 0.072 & 41.354 & 54.327 & $47.092 \mathrm{a} / \mathrm{A}$ & 2.306 & -0.065 & 0.166 & 4.89 \\
\hline & 2013 & $48.333 a / A$ & $46.77 a / A$ & 0.1677 & 42.125 & 51.5 & $46.567 a / A$ & 1.882 & 0.128 & -0.439 & 4.04 \\
\hline \multirow{2}{*}{$\mathrm{ML}_{\mathrm{SL}}$} & 2012 & $47.708 \mathrm{a} / \mathrm{A}$ & $47.083 a / A$ & 1.4245 & 40.781 & 51.458 & $46.581 \mathrm{a} / \mathrm{A}$ & 2.008 & -0.294 & 0.276 & 4.31 \\
\hline & 2013 & $47.604 a / A$ & $45.52 \mathrm{a} / \mathrm{A}$ & 3.355 & 40.5 & 51.5 & $45.806 \mathrm{~b} / \mathrm{B}$ & 1.9 & 0.114 & -0.072 & 4.15 \\
\hline \multirow{2}{*}{$\mathrm{AG}_{\mathrm{SL}}$} & 2012 & $45.104 a / A$ & $47.083 a / A$ & 0.8505 & 39.546 & 50.938 & $45.719 a / A$ & 2.304 & -0.125 & -0.464 & 5.03 \\
\hline & 2013 & $44.583 a / A$ & $44.687 a / A$ & 0.3065 & 40.625 & 49.75 & $45.664 a / A$ & 2.066 & -0.163 & -0.486 & 4.52 \\
\hline \multirow[t]{2}{*}{$\mathrm{MG}_{\mathrm{SL}}$} & 2012 & $47.916 \mathrm{a} / \mathrm{A}$ & $46.354 a / A$ & $23.3720^{*}$ & 39.375 & 52.188 & $46.014 \mathrm{a} / \mathrm{A}$ & 2.227 & -0.07 & -0.203 & 4.85 \\
\hline & 2013 & $45 a / A$ & $46.25 a / A$ & 2.6286 & 40.5 & 51 & $46.082 \mathrm{a} / \mathrm{A}$ & 2.206 & -0.316 & -0.193 & 4.78 \\
\hline \multirow[t]{2}{*}{$\mathrm{DG}_{\mathrm{SL}}$} & 2012 & $48.229 a / A$ & $46.458 \mathrm{a} / \mathrm{A}$ & $82.2725^{\star \star}$ & 38.281 & 50.833 & $45.22 \mathrm{a} / \mathrm{A}$ & 2.56 & -0.106 & -0.32 & $5.67 \%$ \\
\hline & 2013 & $43.645 \mathrm{~b} / \mathrm{A}$ & $46.562 \mathrm{a} / \mathrm{A}$ & 3.544 & 38.125 & 50 & $44.708 \mathrm{a} / \mathrm{A}$ & 2.38 & -0.006 & --0.502 & 5.32 \\
\hline \multirow[t]{2}{*}{$P_{s w}$} & 2012 & $26.25 \mathrm{a} / \mathrm{A}$ & $24.687 a / A$ & 0.661 & 20.833 & 29.25 & $25.64 a / A$ & 1.303 & -0.41 & 1.752 & 5.08 \\
\hline & 2013 & $25.208 a / A$ & 24.791a/A & 3.2167 & 21.477 & 29.75 & $24.603 \mathrm{~b} / \mathrm{B}$ & 1.06 & 1.055 & 4.471 & 4.30 \\
\hline \multirow[t]{2}{*}{$\mathrm{AL}_{\mathrm{sw}}$} & 2012 & $29.479 a / A$ & $28.125 a / A$ & 1 & 23.75 & 33.462 & $27.239 a / A$ & 1.775 & 0.843 & 1.038 & 6.49 \\
\hline & 2013 & $25.416 \mathrm{~b} / \mathrm{A}$ & $25.208 a / A$ & 1.8715 & 23.854 & 30.5 & $25.921 \mathrm{~b} / \mathrm{B}$ & 1.013 & 1.095 & 2.356 & 3.91 \\
\hline \multirow[t]{2}{*}{$\mathrm{ML}_{\mathrm{sw}}$} & 2012 & $26.562 \mathrm{a} / \mathrm{A}$ & $26.25 a / A$ & 0.9116 & 24.063 & 34.583 & $27.062 a / A$ & 1.623 & 1.212 & 2.975 & 5.99 \\
\hline & 2013 & $26.77 \mathrm{a} / \mathrm{A}$ & $25.52 \mathrm{a} / \mathrm{A}$ & 3.6056 & 23.75 & 29.625 & $25.967 \mathrm{~b} / \mathrm{B}$ & 1.067 & 0.748 & 0.663 & 4.11 \\
\hline \multirow[t]{2}{*}{$\mathrm{AG}_{\mathrm{sW}}$} & 2012 & $26.666 a / A$ & $33.75 a / A$ & 0.8505 & 24.375 & 37.789 & $29.133 a / A$ & 2.327 & 0.915 & 1.608 & 7.97 \\
\hline & 2013 & $29.375 a / A$ & $28.125 \mathrm{~b} / \mathrm{A}$ & 0.3065 & 25.125 & 33.5 & $27.662 \mathrm{~b} / \mathrm{B}$ & 1.29 & 1.237 & 2.861 & 4.66 \\
\hline \multirow[t]{2}{*}{$M G_{s w}$} & 2012 & $29.652 a / A$ & $30 a / A$ & $23.372^{*}$ & 25.625 & 38.125 & $29.499 a / A$ & 2.123 & 0.703 & 1.218 & 7.17 \\
\hline & 2013 & $28.229 a / A$ & $28.75 a / A$ & 2.6286 & 24.5 & 32.969 & $27.883 \mathrm{~b} / \mathrm{B}$ & 1.565 & 0.482 & 0.518 & 5.61 \\
\hline \multirow[t]{2}{*}{$\mathrm{DG}_{\mathrm{sw}}$} & 2012 & $30 a / A$ & $30.729 a / A$ & $82.2725^{\star \star}$ & 24.531 & 37.604 & $29.271 \mathrm{a} / \mathrm{A}$ & 2.384 & 0.625 & 0.39 & 8.11 \\
\hline & 2013 & $28.645 a / A$ & $31.562 \mathrm{a} / \mathrm{A}$ & 3.544 & 23.75 & 34.375 & $27.484 \mathrm{~b} / \mathrm{B}$ & 1.868 & 0.97 & 1.437 & 6.77 \\
\hline
\end{tabular}

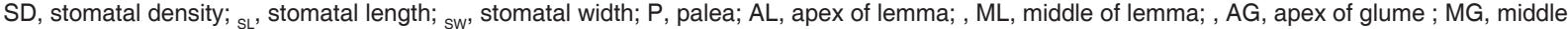
of glume; DG, down of glume; SD, standard deviation; CV, coefficient of variation; * and ** indicate significance at $\mathrm{P}<0.05$ and $\mathrm{P}<0.01$, respectively

the two years were not significant. For DH lines, stomatal densities of the rest parts in 2012 were significantly more than those in 2013, except for apex of lemma and down of glume (Table 1).

Except for down of glume of Hanxuan10, differences of stomatal length for the rest parts of spike organs between the two years were not significant for the two parents. Differences of stomatal length for the rest parts between the two years were also not significant for DH lines, except for palea and middle of lemma (Table 1)

Except for apex of lemma of Hanxuan10 and apex of glume of Lumai14, differences of stomatal width for the rest parts of spike organs between the two years were not significant for the two parents and DH lines (Table 1)

\section{Correlation for stomatal density and size}

For all parts of spike organ, stomatal density showed a highly significant negative correlation with stomatal length in 2012 and 2013. But correlation between stomatal density and stomatal width was not significant. Stomatal length was significant and highly significant positive correlation with stomatal width in 2012, and except for middle of lemma, apex and down of glume in 2013 (Table 2).

\section{Additive QTL for traits related to stomata in wheat spike organ}

Stomatal density and size of spike organs for DHLs showed continuous transgressive segregation with smaller skewed value and kurtosis values (Table 1), suggesting normal distribution. All target traits were thus quantitatively controlled by multiple genes and were suitable for QTL mapping. 
Table 2: Correlation analysis of stomatal density, stomatal length and stomatal width of various parts of spikelet in different years

\begin{tabular}{|c|c|c|c|c|c|c|c|c|c|c|c|c|c|}
\hline Trait & Environment & $\mathbf{P}_{\mathrm{SD}}$ & $\mathrm{AL}_{\mathrm{SD}}$ & $M L_{S D}$ & $\mathrm{AG}_{\mathrm{SD}}$ & $M G_{S D}$ & $\mathrm{DG}_{\mathrm{SD}}$ & $\mathbf{P}_{\mathrm{SL}}$ & $\mathrm{AL}_{\mathrm{SL}}$ & $\mathrm{ML}_{\mathrm{SL}}$ & $\mathrm{AG}_{\mathrm{SL}}$ & $\mathrm{MG}_{\mathrm{SL}}$ & $\mathrm{DG}_{\mathrm{SL}}$ \\
\hline \multirow{2}{*}{$P_{s w}$} & 2012 & $\left.0.09^{2}\right)$ & -0.08 & -0.04 & -0.13 & 0.06 & 0 & $0.18^{\star}$ & $0.19^{*}$ & $0.27^{* *}$ & 0.15 & $0.19^{*}$ & $0.18^{*}$ \\
\hline & 2013 & 0 & $-0.17^{*}$ & -0.04 & -0.16 & -0.14 & -0.05 & $0.41^{* *}$ & $0.21^{* *}$ & $0.17^{\star}$ & 0.07 & $0.23^{* *}$ & $0.24^{* *}$ \\
\hline \multirow[t]{2}{*}{$\mathrm{AL}_{\mathrm{sw}}$} & 2012 & 0.05 & -0.01 & 0.13 & 0.1 & 0.08 & 0 & 0.14 & $0.20^{*}$ & 0.12 & $0.19^{*}$ & 0.14 & $0.18^{*}$ \\
\hline & 2013 & -0.06 & -0.13 & -0.04 & -0.03 & 0.03 & 0 & 0.16 & $0.22^{* *}$ & 0.11 & 0.14 & 0.16 & $0.19^{*}$ \\
\hline \multirow[t]{2}{*}{$\mathrm{ML}_{\mathrm{sw}}$} & 2012 & 0.03 & -0.04 & 0.06 & -0.09 & 0.01 & -0.02 & 0.13 & $0.27^{\star \star}$ & $0.20^{*}$ & $0.27^{\star \star}$ & $0.20^{*}$ & 0.16 \\
\hline & 2013 & -0.02 & -0.11 & 0.06 & -0.03 & 0.06 & 0.06 & $0.27^{* *}$ & $0.18^{*}$ & 0.16 & 0.12 & 0.12 & $0.29^{* *}$ \\
\hline \multirow{2}{*}{$\mathrm{AG}_{\mathrm{sw}}$} & 2012 & 0.15 & -0.02 & 0.11 & -0.09 & 0.1 & 0.09 & 0.13 & $0.26^{\star \star}$ & $0.27^{\star *}$ & $0.17^{\star}$ & $0.21^{*}$ & $0.17^{*}$ \\
\hline & 2013 & 0.07 & -0.13 & 0 & -0.1 & -0.09 & 0.12 & 0.1 & 0.15 & 0.13 & 0.04 & 0.11 & $0.18^{*}$ \\
\hline \multirow[t]{2}{*}{$M G_{s w}$} & 2012 & 0.13 & -0.05 & 0.08 & 0.01 & 0.08 & 0.08 & 0.11 & $0.30^{* *}$ & $0.32^{* *}$ & $0.21^{* *}$ & $0.21^{*}$ & $0.26^{\star *}$ \\
\hline & 2013 & -0.01 & $-0.19^{*}$ & -0.08 & -0.04 & -0.04 & 0.04 & 0.11 & $0.25^{\star *}$ & $0.18^{*}$ & 0.12 & $0.24^{* *}$ & 0.13 \\
\hline \multirow[t]{2}{*}{$D G_{s w}$} & 2012 & $0.19^{*}$ & -0.12 & 0.07 & -0.01 & 0.02 & -0.01 & 0.09 & $0.26^{* *}$ & $0.22^{* *}$ & $0.20^{\star}$ & $0.20^{*}$ & $0.26^{* *}$ \\
\hline & 2013 & 0.09 & -0.06 & 0.13 & -0.09 & 0.03 & 0.01 & -0.06 & 0.08 & 0.04 & 0.03 & 0.13 & 0.05 \\
\hline \multirow[t]{2}{*}{$P_{S L}$} & 2012 & $-0.20^{*}$ & $-0.41^{\star *}$ & $-0.35^{\star *}$ & $-0.46^{\star \star}$ & $-0.33^{\star *}$ & $-0.27^{\star *}$ & & & & & & \\
\hline & 2013 & $-0.30^{\star *}$ & $-0.25^{\star *}$ & $-0.22^{* *}$ & $-0.29^{\star \star}$ & $-0.24^{\star \star}$ & -0.16 & & & & & & \\
\hline \multirow[t]{2}{*}{$\mathrm{AL}_{\mathrm{SL}}$} & 2012 & -0.11 & $-0.48^{\star *}$ & $-0.29^{\star *}$ & $-0.38^{\star *}$ & $-0.33^{\star \star}$ & $-0.22^{\star *}$ & & & & & & \\
\hline & 2013 & $-0.17^{*}$ & $-0.51^{\star *}$ & $-0.18^{*}$ & $-0.29^{\star \star}$ & $-0.27^{\star \star}$ & -0.15 & & & & & & \\
\hline \multirow[t]{2}{*}{$\mathrm{ML}_{\mathrm{SL}}$} & 2012 & $0.19^{*}$ & $-0.34^{\star *}$ & $-0.35^{\star *}$ & $-0.29^{\star \star}$ & $-0.20^{*}$ & $-0.21^{\text {** }}$ & & & & & & \\
\hline & 2013 & $-0.16^{*}$ & $-0.41^{\star *}$ & $-0.43^{* *}$ & $-0.30^{\star \star}$ & $-0.32^{\star *}$ & $-0.31^{* *}$ & & & & & & \\
\hline \multirow[t]{2}{*}{$A G_{S L}$} & 2012 & 0 & $-0.44^{\star *}$ & $-0.33^{\star *}$ & $-0.46^{\star *}$ & $-0.37^{\star *}$ & $-0.34^{* *}$ & & & & & & \\
\hline & 2013 & $-0.20^{*}$ & $-0.53^{\star *}$ & $-0.31^{\star *}$ & $-0.45^{\star *}$ & $-0.43^{\star \star}$ & $-0.27^{\star *}$ & & & & & & \\
\hline \multirow[t]{2}{*}{$\mathrm{MG}_{\mathrm{SL}}$} & 2012 & -0.02 & $-0.50^{\star *}$ & $-0.43^{\star *}$ & $-0.50^{\star *}$ & $-0.51^{\star *}$ & $-0.47^{\star *}$ & & & & & & \\
\hline & 2013 & -0.14 & $-0.39^{* *}$ & $-0.28^{* *}$ & $-0.33^{\star *}$ & $-0.43^{\star *}$ & $-0.41^{* *}$ & & & & & & \\
\hline \multirow[t]{2}{*}{$\mathrm{DG}_{\mathrm{SL}}$} & 2012 & 0 & $-0.37^{\star *}$ & $-0.34^{* *}$ & $-0.40^{\star *}$ & $-0.41^{\star *}$ & $-0.38^{\star *}$ & & & & & & \\
\hline & 2013 & -0.12 & $-0.33^{\star *}$ & $-0.24^{* *}$ & $-0.23^{\star \star}$ & $-0.26^{\star *}$ & $-0.31^{* *}$ & & & & & & \\
\hline
\end{tabular}

SD, stomatal density; , stomatal length; ${ }_{\text {sw }}$, stomatal width; P, palea; AL, apex of lemma; , ML, middle of lemma; , AG, apex of glume ; MG, middle of glume; DG, down of glume; SD, standard deviation; * and ** indicate significance at $\mathrm{P}<0.05$ and $\mathrm{P}<0.01$, respectively

Table 3: Additive effect QTL for stomata-related traits of spike organs in DH population

\begin{tabular}{|c|c|c|c|c|c|c|c|c|c|c|}
\hline \multirow[t]{2}{*}{ Traits } & \multirow[t]{2}{*}{ QTL } & \multirow[t]{2}{*}{ Marker interval } & \multicolumn{4}{|c|}{2012} & \multicolumn{4}{|c|}{2013} \\
\hline & & & Position a & $A^{b}$ & P-value & $\mathrm{H}^{2 \mathrm{c}}$ & Position a & $A^{b}$ & P-value & $\mathrm{H}^{2 \mathrm{c}}$ \\
\hline $\mathrm{P}_{\mathrm{SD}}$ & $Q P_{\mathrm{sd}}-5 B$ & Xgwm371-Xgwm335 & 46.5 & -2.61 & 0.000018 & $11.05 \%$ & & & & \\
\hline \multirow[t]{3}{*}{$\mathrm{AL}_{\mathrm{SD}}$} & $Q A L_{\mathrm{sd}}-5 A-1$ & Xgwm410-WMC340 & 109.5 & 4.99 & 0.000017 & $11.10 \%$ & & & & \\
\hline & $Q A L_{\mathrm{sd}}-5 A-2$ & WMC410-WMC74 & & & & & 21.2 & 2.3037 & 0.000574 & $2.30 \%$ \\
\hline & $Q A L_{s d}-5 D$ & Xgdm68-Xgdm3 & & & & & 11.5 & -2.336 & 0.000463 & $8.35 \%$ \\
\hline $\mathrm{ML}_{\mathrm{SD}}$ & $Q M L_{\mathrm{sd}}-5 A$ & Xgwm291-Xgwm410 & 79.1 & 4.537 & 0.000047 & $10.04 \%$ & 79.1 & 3.9435 & 0.0000 & $15.06 \%$ \\
\hline$A G_{S D}$ & $Q A G_{\mathrm{sd}}-5 A$ & Xgwm410-WMC340 & 109.5 & 5.921 & 0.000058 & $9.86 \%$ & & & & \\
\hline \multirow[t]{2}{*}{$M G_{S D}$} & $Q M G_{s d}-5 A-1$ & WMC410-WMC74 & 37.2 & 4.211 & 0.000027 & $10.48 \%$ & & & & \\
\hline & $Q M G_{s d}-5 A-2$ & Xgwm410-WMC340 & & & & & 109.5 & 6.2509 & 0.0000 & $17.72 \%$ \\
\hline $\mathrm{DG}_{\mathrm{SD}}$ & $Q D G_{s d}-5 A$ & Xgwm291-Xgwm410 & 72.1 & 4.57 & 0.000021 & $10.73 \%$ & 74.1 & 6.1264 & 0.0000 & $19.85 \%$ \\
\hline $\mathrm{ML}_{\mathrm{SL}}$ & $Q M L_{\mathrm{sl}}-5 A$ & P3616.5-P3616.6 & & & & & 54.4 & 0.5265 & 0.001745 & $10.12 \%$ \\
\hline$A G_{S L}$ & $Q A G_{\mathrm{sl}}-5 A$ & Xgwm410-WMC340 & 108.5 & -1.61 & 0.0000 & $15.33 \%$ & 105.5 & -1.121 & 0.000054 & $9.93 \%$ \\
\hline$D G_{S L}$ & $Q D G_{\mathrm{sl}}-3 B$ & P3156.1-P5138 & & & & & 163.4 & 1.1792 & 0.000817 & $6.98 \%$ \\
\hline $\mathrm{ML}_{\mathrm{sw}}$ & $Q M L_{\mathrm{sw}}-2 A$ & Xpsp3088-WMC296 & & & & & 57.4 & -0.58 & 0.000134 & $8.97 \%$ \\
\hline$A G_{S W}$ & $Q A G_{\mathrm{sw}}-4 B$ & WMC47-3459.1 & 121 & -0.96 & 0.000098 & $9.30 \%$ & & & & \\
\hline
\end{tabular}

a Positions (cM) represents the distance to the first marker in the linkage group; ${ }^{\mathrm{b}} \mathrm{A}$ represents the additive effect. Positive value indicates the Hanxuan 10 allele having positive effect on the trait, and negative value represents Lumai 14 allele having positive effect; ${ }^{\circ} \mathrm{H} 2(\mathrm{~A})$ indicates the phenotypic variance explained by additive QTL. ${ }_{\mathrm{SD}}$, stomatal density; sL, stomatal length; ${ }_{\mathrm{sw}}$, stomatal width; P, palea; AL, apex of lemma; , ML, middle of lemma; , AG, apex of glume ; MG, middle of glume; $\mathrm{DG}$, down of glume; $\mathrm{SD}$, standard deviation.

A total of nine additive QTLs for stomatal density of spike organ were detected in two years. Phenotypic variation of these QTLs ranged from 2.30 to $19.85 \%$. Among these QTLs, $Q_{M L s d}-5 A$ and $Q_{D G s d}-5 A$ were detected in 2012 and 2013, with additive effects from favorable alleles of H10. $Q P_{s d}-5 B$, $Q A L_{s d}-5 A-1, Q A G_{s d}-5 A$ and $Q M G_{s d}-5 A-1$ were detected in 2012, the rest three QTLs were mapped in 2013(Table 3).
Three additive QTLs for stomatal length of spike organ were detected in two years with phenotypic variation range from $6.98 \%$ to $15.33 \%$. $Q A G_{s l}-5 A$ detected in 2012 and 2013 were at the same marker region with $Q_{M L s d}-5 A$ and $Q_{D G s d}-5 A$, but with opposite direction of additive effect. The rest two QTLs were mapped in 2013 (Table 3). 


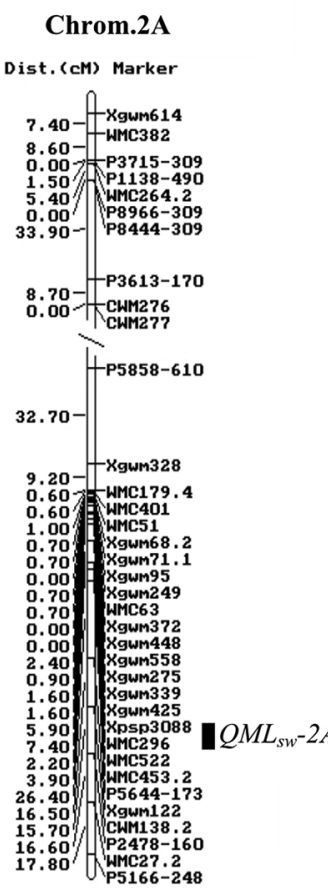

Chrom.4B

Dist. (cM) Marker

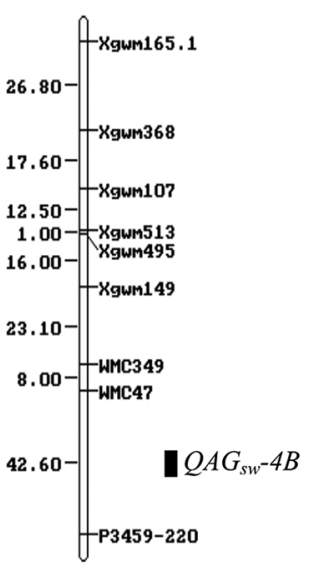

Chrom.5A
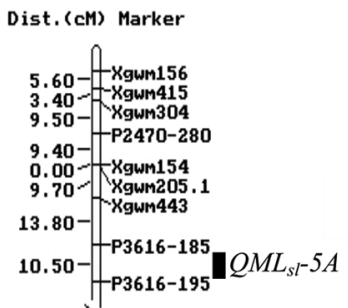

ist. (cM) Marker

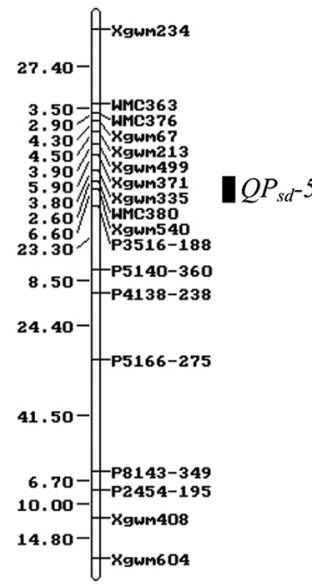

Chrom.3B

Dist. (cM) Marker

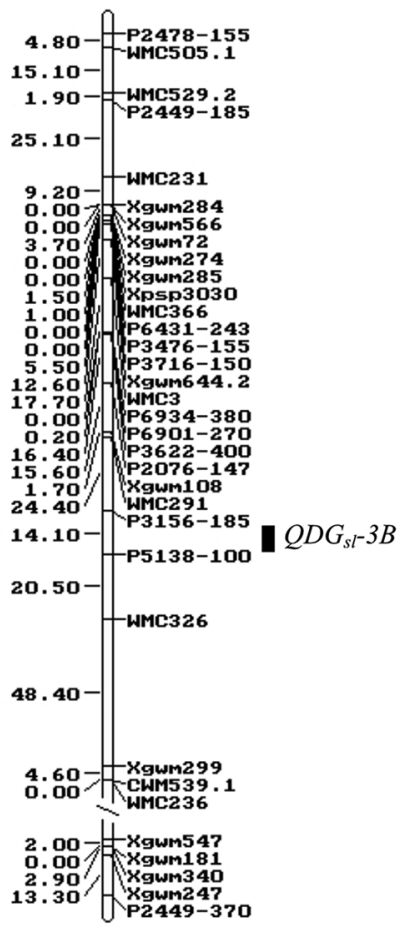

Chrom.5D

Dist. (cM) Marker

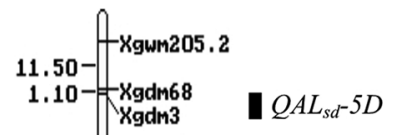

Fig 2. The distribution of QTLs for traits related to the stomata of wheat spike organs on genetic linkage groups constructed on the DH population (produced from the Hanxuan 10xLumai 14 cross).

One additive QTLs for stomatal width of spike organ were detected in 2012 and 2013, respectively (Table 3).

\section{DISCUSSION}

Stomatal density and size in rice leaves are strongly negatively correlated (Ishihara et al., 1979; Ishimaru et al., 2001a; Ohsumi et al. 2007). Stomatal density was also negatively correlated with stomatal length under different water conditions in jujube (Liu et al., 2006) and Platanus acerifolia leaves (Zhang et al., 2004). The present study found stomatal density showed a highly significantly negative correlation with stomatal length for all parts of spike organ in 2012 and 2013. But correlation between stomatal density and stomatal width was not significant. Stomatal length was significantly or highly significantly positive correlation with stomatal width in 2012, and except for middle of lemma, apex and down of glume in 2013. In the previous study, we found that stomatal density of wheat leaf was always significantly and negatively correlated with stomatal 
length at the heading, flowering, and mid- and late grain filling stage, but a negative relationship between stomatal density and width was significant only at heading under well-watered condition and at flowering and late grain filling under drought stress. Stomatal length and width of wheat leaf showed positive and significant correlations, except at heading under drought stress and flowering under well-watered (Wang et al., 2016). Therefore, all green organs of plant can improve their adaptability to different environment conditions through co-ordinated or compensatory variation in stomatal density and length, stomatal density and width, or stomatal length and width. On the other hands, stomatal density and width of wheat spike organs in 2012 were more than those in 2013, but the difference of stomatal length between the two years was not significant. These results indicated that stomatal density and width may have higher plasticity than stomatal length in response to different environments.

Tanksley et al. (1996) thoughtthat main effect QTL can explain more than $10 \%$ of the phenotypic variation. In this study, a total of fourteen additive QTLs were detected for traits related to stomata of spike organs. Eight of these QTLs explained more than 10\% of the phenotypic variation, suggesting that all target traits were quantitative and co-controlled by major and minor genes. In the present study, among the nine additive QTLs for stomatal density, seven increasing stomatal density were alleles from H10. Except for $Q A G_{s l}-5 A$, the rest two additive QTLs increasing stomatal length were also from H10 alleles. But two additive QTLs increasing stomatal width were from L14 alleles (Table 3). The findings confirmed large genetic differences existed between the two parents.

Various studies have found that QTLs for closely correlated traits may be located at, or near, the same chromosomal positions (Hervé et al., 2001; Fracheboud et al., 2002; Tuberosa et al., 2002). Phenotypic correlations between stomatal density and length were found in our work and in previous studies (Zhang et al., 2004; Wang et al., 2016). In the present study, there were $Q_{M L s d}-5 A$ for stomatal density at middle of lemma and $Q_{D G s d}-5 A$ for stomatal density at down of glume, and $Q_{A G S l}-5 A$ for stomatal length at apex of glume were detected on the marker interval Xgwm291-Xgwm410-WMC340 on chromosome 5A in 2012 and 2013, but with opposite direction of additive effect. Previously, $Q s d-5 A .3$ and $Q s d-5 A .4$ for stomatal density of wheat leaf, and $Q s l-5 A .1$ for stomatal length of wheat leaf were also mapped at the same marker region, similarly with opposite direction of additive effect (Wang et al., 2016). These findings implied stomatal density and length for wheat leaf and spike organs may be governed by the same or pleiotropic genes, and once again provided the molecular basis for close correlation between stomatal density and length.

\section{ACKNOWLEDGEMENTS}

This work was supported by National Key R\&D Program of China (2017YFD0300202), the National Science and Technology Major Projects for Cultivation of New Transgenic Varieties (2014ZX0800203B-003).

\section{Author's contributions}

Shuguang Wang and Fanfan Dong conducted the experiment, analyzed the results, discussed the results, and drafted the manuscript. Daizhen Sun supervised and designed the research. Yaoyu Chen and Xue Yan analyzed the results and made figures. Ruilian Jing provided materials and reviewed the manuscript.

\section{REFERENCES}

Fracheboud, Y., J. M. Ribaut, M. Vargas and R. Mesame. 2002. Identification of quantitative trait loci for cold tolerance of photosynthesis in maize (Zea mays L.). J. Exp. Bot. 53: 1967-1977.

Hervé, D., F. Francoise, F. B. Ericka, L. Nadia, A. C. Ghias, P. Claude, S. Ahmad and G. Laurent. 2001. QTL analysis of photosynthesis and water status traits in sunflower (Helianthus annuus L.) under greenhouse condition. J. Exp. Bot. 52: 1857-1864.

Ishihara, K., O. lida and T. Hirasawa. 1979. Relationship between nitrogen content in leaf blades and photosynthetic rate of rice plants with reference to stomatal aperture and conductance. Jpn. J. Crop Sci. 48: 551-556.

Ishimaru, K., K. Shirota, M. Higa and Y. Kawamitsu. 2001a. Identification of quantitative trait loci for adaxial and abaxial stomatal frequencies in Oryza sativa. Plant Physiol. Bioch. 39: 173-177.

Jing, R. L., X. P. Chang, J. Z. Jia and R. H. Hu. 1999. Establishing wheat doubled haploid population for genetic mapping by anther culture. Biotechnology. 9: 4-8.

Laza, M. R. C., M. Kondo, O. Ideta, E. Barlaan and T. Imbe. 2010. Quantitative trait loci for stomatal density and size in lowland rice. Euphytica. 172: 149-158.

Liu, S. P., J. M. Liu, J. Y. Cao, C. Y. Bai and Y. Shi. 2006. Stomatal distribution and character analysis of leaf epidermis of jujube under drought stress. J. Anhui Agric. Sci. 34: 1315-1318.

Meidner, H. 1986. Cuticular conductance and the humidity response of stomata. J. Exp. Bot. 37: 517-525.

Ohsumi, A., T. Kanemura, K. Homma, T. Horie and T. Shiraiwa. 2007. Genotypic variation of stomatal conductance in relation to stomatal density and length in rice (Oryza sativa L.). Plant Prod. Sci. 10: 322-328.

Tanksley, S. D. and J. C. Nelson. 1996. Advanced backcross QTL analysis: A method for the simultaneous discovery and transfer of valuable QTLs from unadapted germplasm into elite breeding lines. Theor. Appl. Genet. 92: 191-203.

Teare, I. D., C. J. Peretson and A. G. Law. 1971. Size and frequency of leaf stomata in cultivars of Triticum aestivum and other Triticum species. Crop Sci. 8: 496-498. 
Tuberosa, R., S. Salvi, M. C. Sanguineti, P. Landi, M. Maccaferri and S. Conti. 2002. Mapping QTL regulating morphophysiological traits and yield: Case studies, shortcomings and perspectives in drought stressed maize. Ann. Bot. 89: 941-963.

Wang, S. G., S. S. Jia, D. Z. Sun, H. Fan, X. P. Chang and R. L. Jing. 2016. Mapping QTLs for stomatal density and size under drought stress in wheat (Triticum aestivum L.). J. Integr. Ag. 9: 1955-1967.

Zhang, H., X. Y. Wang and S. B. Wang. 2004. A study on stomatal traits of Platanus acerifolia under urban stress. J. Fudan Univ. 43: 651-656.
Zhang, Y. P, Z. M. Wang, Y. C. Wu and X. Zhang. 2006. Stomatal characteristics of different green organs in wheat under different irrigation regimes. Acta Agron. Sin. 32: 70-75.

Zhang, Y. P., Z. M. Wang, Q. Huang and M. Xie. 2008. Changes of chloroplast ultramicrostructure and function of different green organs in wheat under limited irrigation. Acta Agron. Sin. 34: 1213-1219.

Ziegler-Jons, A. 1989. Gas exchange of ears of cereals in response to carbon dioxide and light: II. occurrence of a $\mathrm{C}_{3}-\mathrm{C}_{4}$ intermediate type of photosynthesis. Planta. 178: 164-175.

\section{SUPPLEMENTARY}

Supplementary Table 1: SSR marker sequences linked with QTLs detected in the study

\begin{tabular}{lll}
\hline Marker & Forward primer & Reverse primer \\
\hline Xgwm371 & GACCAAGATATTCAAACTGGCC & AGCTCAGCTTGCTTGGTACC \\
Xgwm335 & CGTACTCCACTCCACACGG & CGGTCCAAGTGCTACCTTTC \\
Xgwm410 & GCTTGAGACCGGCACAGT & CGAGACCTTGAGGGTCTAGA \\
WMC410 & GGACTTGAAAGGAAGCTTGTGA & CATGGATGGCATGCAGTGT \\
WMC74 & AACGGCATTGAGCTCACCTTGG \\
Xgdm68 & GCCTGACCACTCCCATAAAA & TGCGTGAAGGCAGCTCAATCGG \\
Xgdm3 & GTATCTCGGTGATGCAGCAA & TCGGAAGGGGGACTATACAA \\
Xgwm291 & CATCCCTACGCCACTCTGC & GTGTGATGTTTGAATACGCA \\
Xpsp3088 & AATGGTATCTATTCCGACCCG \\
WMC296 & GTGGTGTTACTTTTAGGTTTCTCC & GGACCATTGGTATGTTTTCTAGTC \\
WMC47 & GAATCTCATCTTCCCTTGCCAC & ATGGAGGGGTATAAAGACAGCG \\
\hline
\end{tabular}

